\title{
Gynecologists' attitudes regarding human papilloma virus vaccination: a survey of Fellows of the American College of Obstetricians and Gynecologists
}

\author{
Janice C. Raley ${ }^{1}$, Kristen A. Followwill ${ }^{1}$, Gregorgy D. Zimet ${ }^{4}$ and Kevin A. Ault ${ }^{1,2,3}$ \\ ${ }^{1}$ Department of Obstetrics and Gynecology, University of Iowa, Iowa City, IA, USA \\ ${ }^{2}$ Department of Epidemiology, \\ ${ }^{3}$ Holden Comprehensive Cancer Center, University of Iowa, Iowa City, IA, USA \\ ${ }^{4}$ Department of Pediatrics, Indiana University School of Medicine, Indianapolis, IN, USA
}

Background: Human papilloma virus (HPV) is the causative agent of cervical neoplasia and genital warts. A vaccine has recently been developed that may prevent infection with HPV. Vaccination for HPV may become a routine part of office gynecology. We surveyed members of the American College of Obstetricians and Gynecologists (ACOG) to determine their attitudes to HPV vaccination.

Methods: A survey was sent to Fellows of ACOG to evaluate gynecologists' attitudes. Vaccine acceptability was analyzed using 13 scenarios with the following dimensions and respective attributes: age of patient $(3,17$ and 22 years); efficacy of vaccine (50\% or $80 \%$ ); ACOG recommendation (yes or no); and disease targeted (cervical cancer, warts or both). Each scenario was rated by means of an II-point response format (0 to I00). Responses were evaluated using conjoint analysis.

Results: Of 1200surveys that were sent out, 181 were returned and included in our analysis. ACOG recommendation was considered the most important variable in vaccine distribution (importance score $=32.2$ ), followed by efficacy (24.5), age (22.4) and, lastly, disease targeted (20.9). Of these variables, higher efficacy was favored; preference was given to age 17 years, with a strong disinclination to vaccinate at age 13 years; and protection against cervical cancer, or genital warts, or both, was significantly favored over a vaccine against genital warts alone. Demographic characteristics of the gynecologists (i.e., age of physician, gender, practice setting and community size) did not play an important role in the decision to recommend vaccination.

Conclusion: Professional society recommendation is important for acceptability of a potential HPV vaccine. Gynecologists are willing to include this vaccine in their office practice.

Key words: Human Papilloma Virus; Cervical Neoplasia; Vaccine; Sexually Transmitted Diseases; ADOLESCENCE

\section{INTRODUCTION}

Human papilloma virus (HPV) infections are common, with a prevalence of 10 to $20 \%$ in the general population(1) and up to $46 \%$ in university students(2,3). It has been found that virtually all cervical cancers are associated with HPV infection(4,5), 50\% being caused by HPV-16(5). The prevalence of HPV varies with age, with the highest prevalence in the age range 20 to 30

Correspondence to: Kevin A. Ault, MD, Department of Obstetrics and Gynecology, University of Iowa Hospitals and Clinics, 200 Hawkins Drive, Iowa City, IA 52242, USA. Email: kevin-ault@uiowa.edu

(C) 2004 Parthenon Publishing. A member of the Taylor \& Francis Group Ltd.

DOI: 10.1080/I064744040002066 I 
years $(3,6)$. Vaccines to strains of HPV are being developed and studied in human subjects.

One of the vaccines developed uses virus-like particles (VLPs) that are synthetically produced L1 gene proteins which form an empty capsid. The L1 gene is part of the HPV genome which encodes for a protein that self-assembles into a viral capsid which is immunogenic when available to the immune system(7). The vaccine has VLPs, or empty capsids, which are presented as conformational epitopes which induce an immune response greater than do live virons $(7,8)$. In 2002 , it was reported that a vaccine composed of HPV 16 L1 VLPs reduced the prevalence of both HPV 16 infection and related cervical intraepithelial neoplasia. The estimated efficacy of this vaccine was 90 to $100 \%(9)$.

As vaccines are developed for clinical practice, the willingness of physicians to recommend HPV vaccination will be one essential element for the successful implementation of HPV immunization programs. This study examines the acceptability to gynecologists of HPV vaccination for their patients as a function of physician preferences for key vaccine-related characteristics.

\section{METHODS}

A survey was distributed to a random subset of 1200 Fellows of the American College of Obstetricians and Gynecologists (ACOG) throughout the USA. This subset was graciously provided by the research office of ACOG. The survey included 13 scenarios to evaluate vaccine acceptability with regard to the following four dimensions and respective attributes: ACOG approval (yes or no); vaccine efficacy $(50 \%$ or $80 \%)$; age of patient $(13,17$ or 22 years); and targeted disease (cervical cancer, genital warts or both).

For example, the first item read as follows: "Scenario 1: The patient is a 13-year-old girl and is not sexually active. The vaccine is effective against HPV types that cause both cervical cancer and genital warts. Efficacy of the vaccine is $80 \%$. The American College of Obstetricians and Gynecologists (ACOG) has recommended this vaccine be given."
All 13 scenarios are shown in the Appendix. An 11-point scale was used to access physician willingness to recommend vaccination to the patient in each scenario. The scale ranged from 0 (never recommend), through 50 (unsure), to 100 (definitely recommend) in increments of 10 . The physician was asked to evaluate each scenario and circle the number that indicated the likelihood of recommending the vaccine described to the patient. Demographic data were collected, including physician age, gender, community setting, practice setting, year of residency and clinic visits used to treat genital warts and cervical dysplasia.

This study involved four dimensions with two to three attributes for each dimension. To list every possible scenario in the survey, 36 vaccine scenarios would have been required. A subset of nine scenarios was developed using a fractional factorial design through the SPSS Conjoint procedure(10). This method allowed the development of a conjoint model which predicted responses to every vaccine scenario based on the smaller subset. This statistical method was used to quantify the importance of each dimension to the vaccine acceptability as a whole. The limitation of this model is that the interactions of dimensions with one another cannot be evaluated. Conjoint analytic methods increasingly have been applied to research on medical decision-making issues, including acceptability of vaccination $(11,12)$.

Four hold-out scenarios were also developed by the conjoint procedure in order to assess the fit of the conjoint model. Thus, these four items were not used in the development of the conjoint model, but were used to evaluate how closely the model predicted the actual scores associated with those items. The association between the actual scores and the model-predicted scores could be assessed using a Pearson correlation.

The mean rating of all 13 scenarios assessed overall vaccine acceptability. Full-profile, ratingsbased conjoint analysis was used for the statistical analysis. Part-worth utilities were calculated reflecting the physicians' relative preferences for the attributes within a given dimension, e.g. a recommendation from ACOG versus no recommendation from ACOG. A more positive partworth utility score would indicate that the given answer was more highly valued. The relative 
ranges of the part-worth utilities were then converted into importance scores indicating which of the four dimensions were viewed as more significant in vaccine recommendation. In this statistical method, the sum of all importance scores always equals 100 . The highest importance score in the series of four dimensions indicates that this is overall the most valued dimension.

The institutional review board of the University of Iowa approved this survey and research protocol.

\section{RESULTS}

In all 207 gynecologists returned the survey; 26 respondents assigned the same rating to all of the nine scenarios, including 21 who gave a rating of 100 to all nine scenarios. Because these ratings did not vary across scenarios, preferences based on scenario dimensions could not be evaluated. As a result, their surveys could not be used in the conjoint analysis. Therefore, 181 respondent surveys were used to develop our conjoint model. A conjoint model fit was calculated by Pearson correlations between the actual respondent's ratings of 4 hold-out conjoint scenarios and the predicted value of those scenarios based on the conjoint model. The median Pearson correlation was 0.85 , indicating a very good fit for the conjoint model. The demographics for the responders are included in Table I. The overall scenario ratings were not significantly associated with any of the demographic information about the respondents. Across all scenarios used in the model, the mean acceptance of vaccination was 79.0, standard deviation 16.6 (scale of 0 to 100 ).
The part-worth utilities showing physician preference for each attribute were calculated and are displayed in Figure 1A and B. In Figure 1A, gynecologists answering our survey strongly preferred an ACOG recommendation and a more efficacious vaccine. As shown in $1 \mathrm{~B}$, the survey respondents were disinclined to vaccinate 13year-olds. As also shown in this figure, the respondents preferred a vaccine that was directed against HPV types associated with cervical neoplasia and genital warts or a vaccine against oncogenic HPV types only. There was less acceptance of a vaccine targeting only HPV types causing genital warts.

The relative ranges for the part-worth utilities were converted into importance scores and are displayed in Figure 2. ACOG approval contributed most strongly to ratings influencing physician recommendation, with an importance score of 32.2, followed by vaccine efficacy, patient age and vaccine type.

\section{DISCUSSION}

The purpose of our study was to examine how vaccine-related characteristics influenced gynecologists' acceptance of HPV vaccination for patients. The characteristics in which we were interested included ACOG approval, vaccine efficacy, age of patient and disease targeted in the vaccine.

Our study found that the professional organization's recommendation was the variable that most influenced the gynecologists' choice whether to recommend the vaccine to the patient. This finding echoes the results of a recent

Table I Demographic characteristics for the survey respondents

\begin{tabular}{lccc}
\hline Characteristic & \multicolumn{3}{c}{ Values } \\
\hline Age in years & Mean 47.1 & Range $30-72$ & SD I0.7 \\
Years in practice & Mean 16.2 & Range I-42 & SD 28.1 \\
Gender & $55 \%$ male & $45 \%$ female & I5\% rural \\
Work setting & $28 \%$ urban & $56 \%$ suburban & $18.5 \%$, multispecialty \\
Practice type & $62 \%$ solo & $19.5 \%$, teaching & $\mid 4 \%$ small,** \\
Community size & $34 \%$ large* & $30 \%$ mod $\dagger$, & $1 \%$ rural $\dagger \dagger$ \\
\hline
\end{tabular}

*Large city, population more than 500000 ; †moderate city, population 100000 to 499999 ; $\$$ city, population 25000 to 100000 ; **small town, population 5000 to 24999 ; ††rural, population less than 4999. 

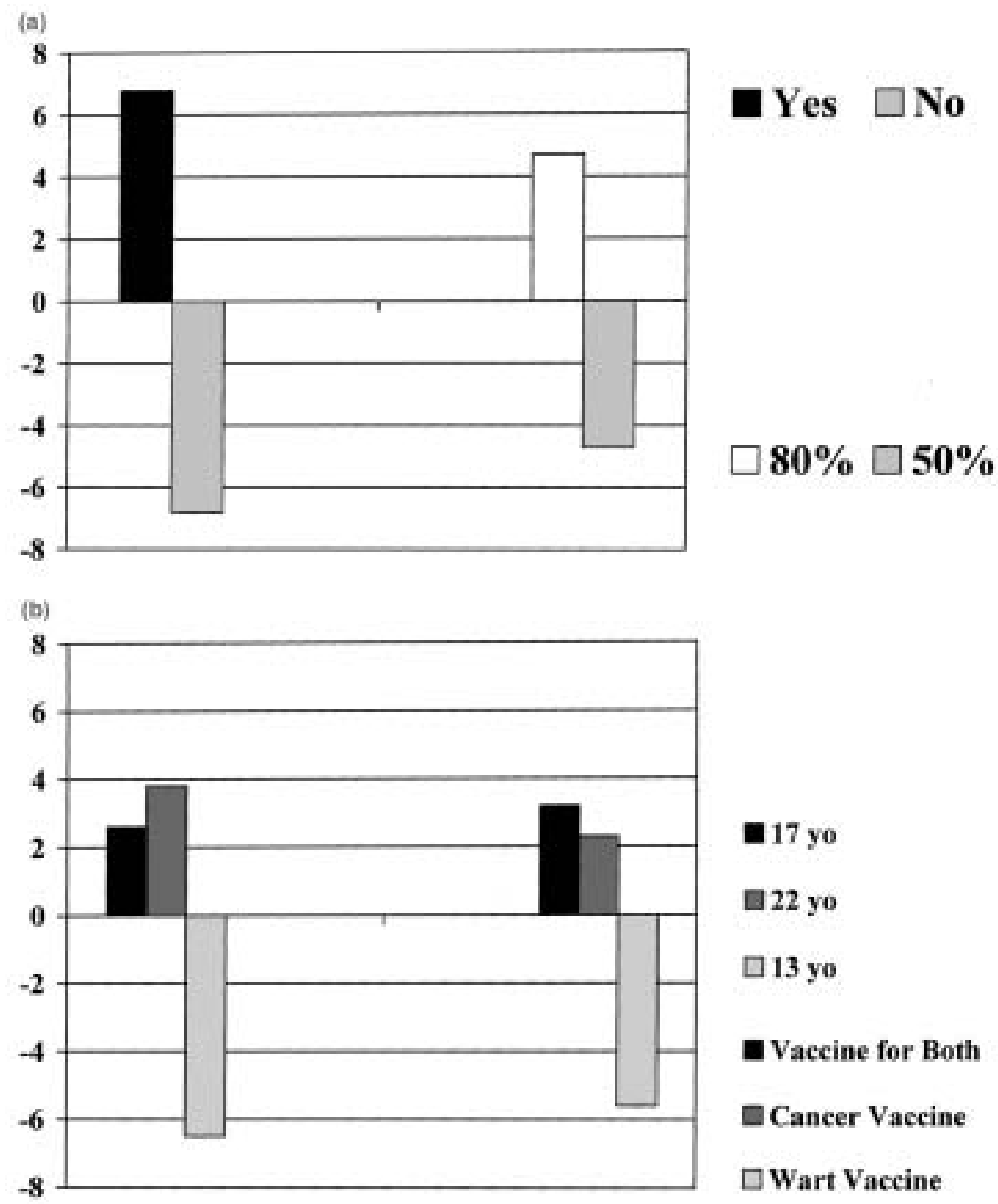

Figure IA and B Part-worth utilities. Higher positive scores indicate more acceptance of that attribute or answer. More negative scores indicate less acceptance

study of nurse practitioners' attitudes to STD vaccination, in which endorsement by the American Academy of Pediatrics was a very important determinant of nurses' willingness to recommend immunization $(11,12)$. This trend was also documented when universal hepatitis B immunization was recommended. Freed et al. recorded that although $82 \%$ of pediatricians knew of the recommendation by the Committee on Immunization Practice for Hepatitis B Vaccination, only $37 \%$ agreed that this was warranted.
After the American Academy of Pediatrics and the American Academy of Family Physicians gave their recommendations for vaccination, $66 \%$ of pediatricians agreed universal immunization was warranted(13). The recommendation of HPV vaccination by ACOG as well as other professional peer groups will be very important to a successful vaccination program.

We found it most interesting that the gynecologists were disinclined to vaccinate 13year-olds, a finding also reported in the nurse 


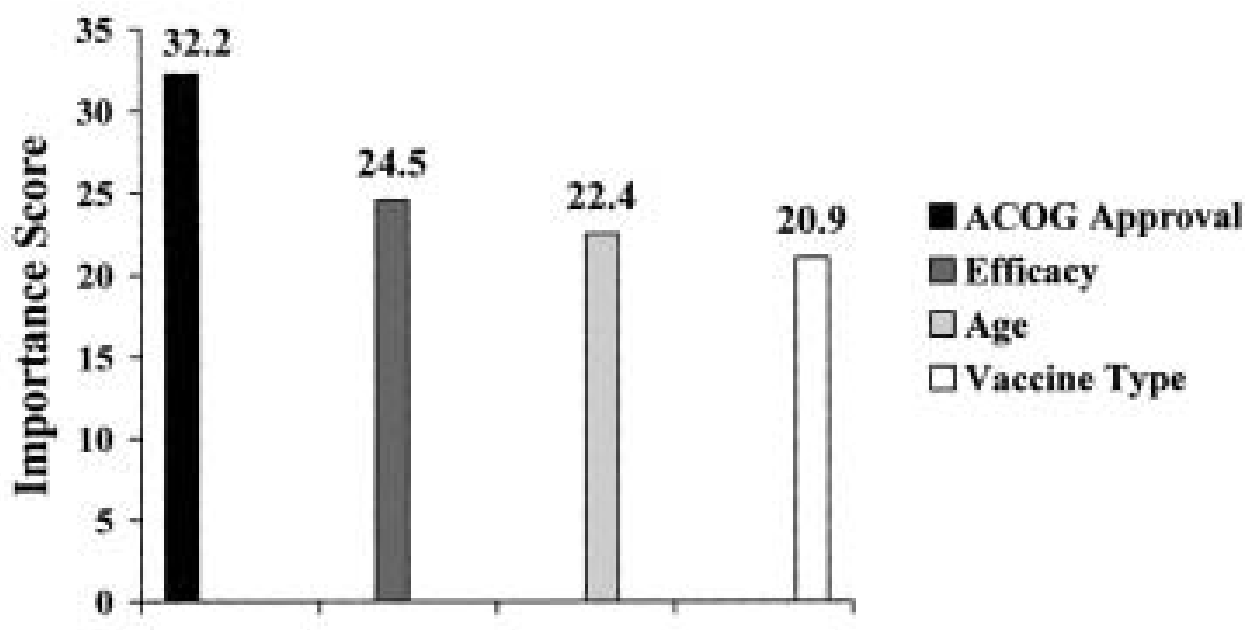

Figure 2 Importance scores. The highest score indicates that this characteristic was most influential in respondents' ratings of the vaccine scenarios

practitioner study. The HPV virus is so common that the chances of getting the infection after one sexual contact are high. Silins et al. reported a correlation between lifetime sexual partners and the prevalence of seropositivity for HPV in Swedish females who had a mean age of 26 years(14). They found that the prevalence of seropositivity rose with the number of sexual partners, from 10\% with one partner, and $25 \%$ with two or three partners and to $53 \%$ with more than 6 partners. Another study reported that each additional sexual partner added about $10 \%$ to the risk of HPV infection(15). Thus the prevalence of infection rapidly increases with the number of partners. Because of the early onset of sexual activity, acceptance of multiple sexual partners and cervical immaturity, 13-year-olds might be the best candidates for a prophylactic HPV vaccine. However, this was the age group for which the vaccine was least likely to be recommended by the gynecologists surveyed. It will be important to include physician education as an essential part of a vaccine implementation program in the offices of gynecologists and other practitioners who care for adolescent women.

The major limitation of this study was the low response rate of $17.3 \%$. In an attempt to maximize our response rate, we devized a short survey; preliminary survey respondents reported that this took 5 to 10 minutes to complete. We also sent a second reminder letter. Our survey respondents were anonymous and were all physicians. In other studies these characteristics decreased the respondence rate by 14 and $9 \%$, respectively(16). A meta-analysis of physician surveys found little difference in demographic variables among early responders, respondents to subsequent mailings, and late responders. This was thought to be because physicians are a more homogeneous group with regard to knowledge, attitudes and behavior. The analysis concluded that a nonresponse bias might not be as critical to interpretation of data gathered from physician surveys with low response rates compared with the general population(17).

Our findings show that an HPV vaccine will be widely accepted (79.0\%) for use by gynecologists. The results also point to the importance of ACOG approval in future HPV immunization practices as well as high vaccine efficacy. Physicians seem to approve more of a vaccine that can combat both types of targeted disease rather than warts alone. Physicians appeared to be uncomfortable about immunizing 13-year-olds, although this may be an excellent age group to target in the prevention of HPV infections. We feel confident that gynecologists will accept an HPV vaccine as part of their routine gynecological practice. 


\section{Acknowledgments}

We are indebted to Ralph Hale, Jay Schulkin and Lori Gentile of the American College of Obstetricians and Gynecologists for their help with this survey. These data were presented in part at the International Infectious Disease Society for Obstetrics and Gynecology USA meeting in New Orleans, LA, USA on April 29, 2003.

\section{REFERENCES}

1. Koutsky L. Epidemiology of genital human papillomavirus infection. Am J Med 1997; 102(5A):3-8

2. Bauer HM, Ting Y, Greer CE, et al. Genital human papillomavirus infection in female university students as determined by a PCR-based method. JAMA 1991;265(4):472-7

3. Sellors JW, Mahony JB, Kaczorowski J, et al. Prevalence and predictors of human papillomavirus infection in women in Ontario, Canada: Survey of HPV in Ontario Women (SHOW) Group. CMAJ 2000;163(5):503-8

4. Walboomers JM, Jacobs MV, Manos MM, et al. Human papillomavirus is a necessary cause of invasive cervical cancer worldwide. J Pathol 1999; 189(1):12-19

5. Bosch FX, Manos MM, Munoz N, et al. Prevalence of human papillomavirus in cervical cancer: a worldwide perspective: International Biological Study on Cervical Cancer (IBSCC) Study Group. J Natl Cancer Inst 1995;87(11):796802

6. Stone KM, Karem KL, Sternberg MR, et al. Seroprevalence of human papillomavirus type 16 infection in the United States. I Infect Dis 2002; 186(10):1396-402

7. Harro CD, Pang YY, Roden RB, et al. Safety and immunogenicity trial in adult volunteers of a human papillomavirus 16 L1 virus-like particle vaccine. J Natl Cancer Inst 2001;93(4):284-92

8. Schiller JT, Lowy DR. Papillomavirus-like particles and HPV vaccine development. Semin Cancer Biol 1996;7(6):373-82
9. Koutsky LA, Ault KA, Wheeler CM, et al. A controlled trial of a human papillomavirus type 16 vaccine. N Engl J Med 2002;347(21):1645-51

10. SPSS Conjoint 8.0. Chicago, IL: SPSS; 1997.

11. Mays R, Strum L, Zimet G. Parental perspectives on vaccinating children against sexually transmitted infections. Soc Sci Med 2004;58:1405-13

12. Mays R, Zimet G. Recommending STI Vaccination to parents of adolescents: the attitudes of nurse practitioners. Sex Transm Dis 2004;31: 428-32

13. Freed GL, Bordley WC, Clark SJ, Konrad TR. Universal hepatitis B immunization of infants: reactions of pediatricians and family physicians over time. Pediatrics 1994;93(5):747-51

14. Silins I, Kallings I, Dillner J. Correlates of the spread of human papillomavirus infection. Cancer Epidemiol Biomarkers Prev 2000;9(9):953-9

15. Shew ML, Fortenberry JD, Miles P, Amortegui AJ. Interval between menarche and first sexual intercourse, related to risk of human papillomavirus infection. J Pediatr 1994;125(4):661-6

16. Asch DA, Jedrziewski MK, Christakis NA. Response rates to mail surveys published in medical journals. J Clin Epidemiol 1997;50(10): 1129-36

17. Kellerman SE, Herold J. Physician response to surveys. A review of the literature. Am J Prev Med 2001;20(1):61-7 


\section{APPENDIX}

Scenario 1: The patient is a 13-year-old girl and is not sexually active. The vaccine is effective against HPV types that cause both cervical cancer and genital warts. Efficacy of the vaccine is $80 \%$. The American College of Obstetricians and Gynecologists (ACOG) has recommended that this vaccine be given.

Scenario 2: The patient is a 13-year-old girl and is not sexually active. The vaccine is effective against HPV types that cause both cervical cancer and genital warts. Efficacy of the vaccine is $80 \%$. ACOG has not recommended for or against administration of this vaccine.

Scenario 3: The patient is a 22-year-old woman with three lifetime sexual partners. The vaccine is effective against HPV types that cause cervical cancer. Efficacy of the vaccine is $80 \%$. ACOG has not recommended for or against administration of this vaccine.

Scenario 4: The patient is a 22-year-old woman with three lifetime sexual partners. The vaccine is effective against HPV types that cause both cervical cancer and genital warts. Efficacy of the vaccine is $50 \%$. ACOG has not recommended for or against administration of this vaccine.

Scenario 5: The patient is a 13-year-old girl and is not sexually active. The vaccine is effective against HPV types that cause cervical cancer. Efficacy of the vaccine is $80 \%$. ACOG has not recommended for or against administration of this vaccine.

Scenario 6: The patient is a 17 -year-old girl and is not sexually active but is seeking contraception. The vaccine is effective against HPV types that cause cervical cancer. Efficacy of the vaccine is $50 \%$. ACOG has recommended that this vaccine be given.

Scenario 7: The patient is a 22-year-old woman with three lifetime sexual partners. The vaccine is effective against HPV types that cause genital warts. Efficacy of the vaccine is $50 \%$. ACOG has recommended that this vaccine be given.

Scenario 8: The patient is a 17-year-old girl and is not sexually active but is seeking contraception. The vaccine is effective against HPV types that cause both cervical cancer and genital warts. Efficacy of the vaccine is $80 \%$. ACOG has recommended that this vaccine be given.

Scenario 9: The patient is a 17 -year-old girl and is not sexually active but is seeking contraception. The vaccine is effective against HPV types that cause genital warts. Efficacy of the vaccine is $80 \%$. ACOG has not recommended for or against administration of this vaccine.

Scenario 10: The patient is a 13 -year-old girl and is not sexually active. The vaccine is effective against HPV types that cause genital warts. Efficacy of the vaccine is $80 \%$. ACOG has recommended that this vaccine be given.

Scenario 11: The patient is a 22-year-old woman with three lifetime sexual partners. The vaccine is effective against HPV types that cause both cervical cancer and genital warts. Efficacy of the vaccine is $50 \%$. ACOG has recommended that this vaccine be given.

Scenario 12: The patient is a 13 -year-old girl and is not sexually active. The vaccine is effective against HPV types that cause cervical cancer. Efficacy of the vaccine is $80 \%$. ACOG has recommended that this vaccine be given.

Scenario 13: The patient is a 22-year-old woman with three lifetime sexual partners. The vaccine is effective against HPV types that cause both cervical cancer and genital warts. Efficacy of the vaccine is $80 \%$. ACOG has recommended that this vaccine be given. 


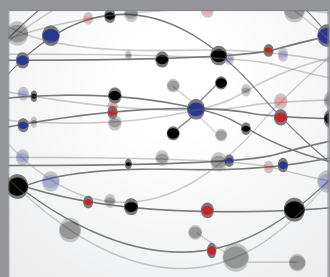

The Scientific World Journal
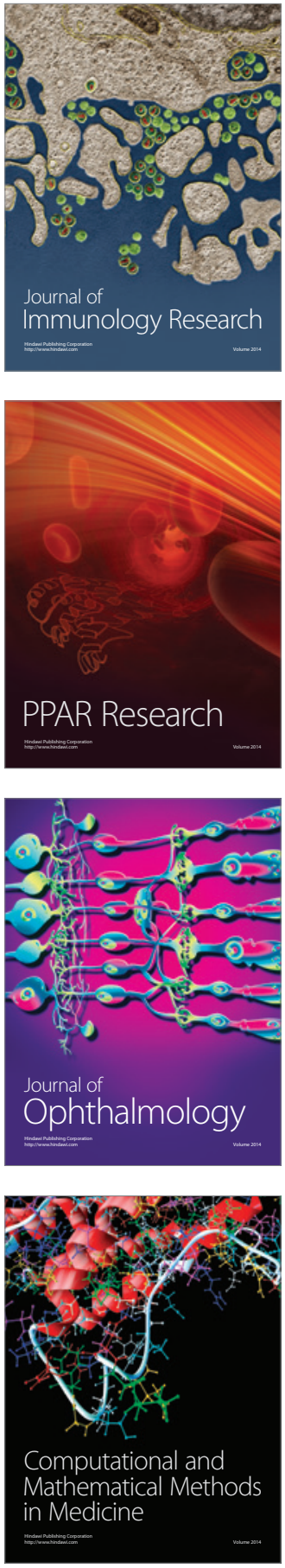

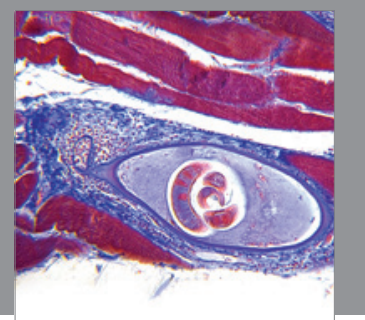

Gastroenterology

Research and Practice
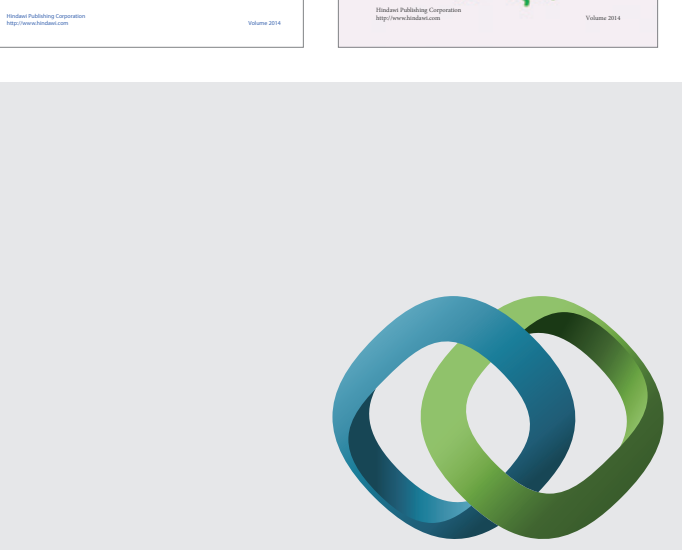

\section{Hindawi}

Submit your manuscripts at

http://www.hindawi.com
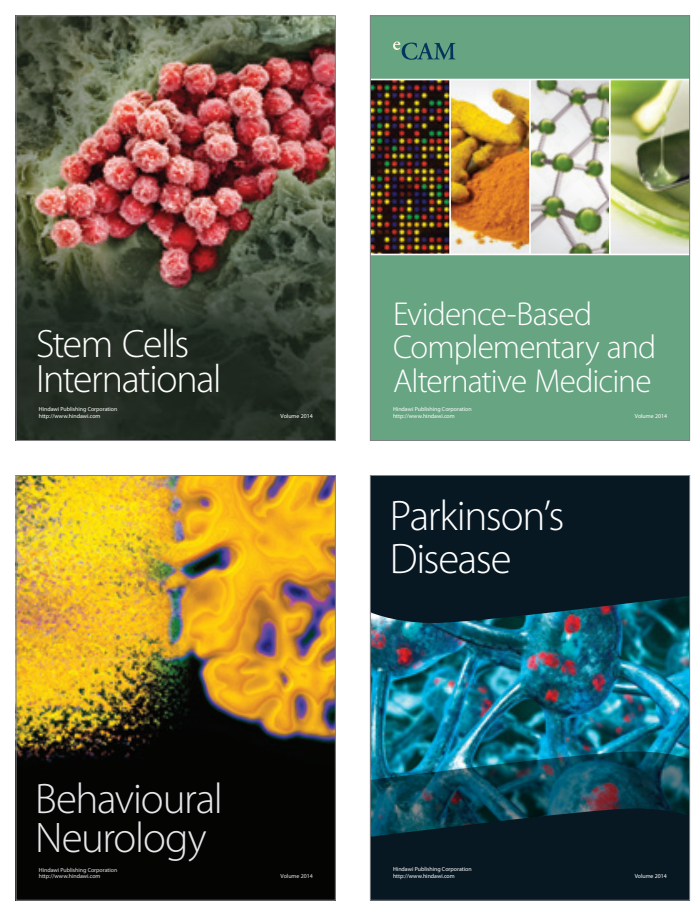

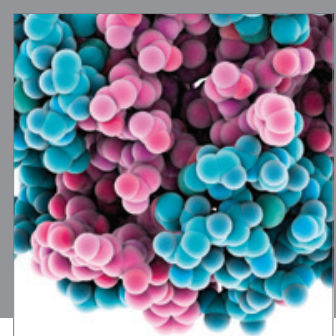

Journal of
Diabetes Research

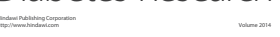

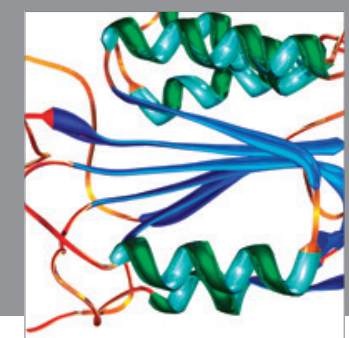

Disease Markers
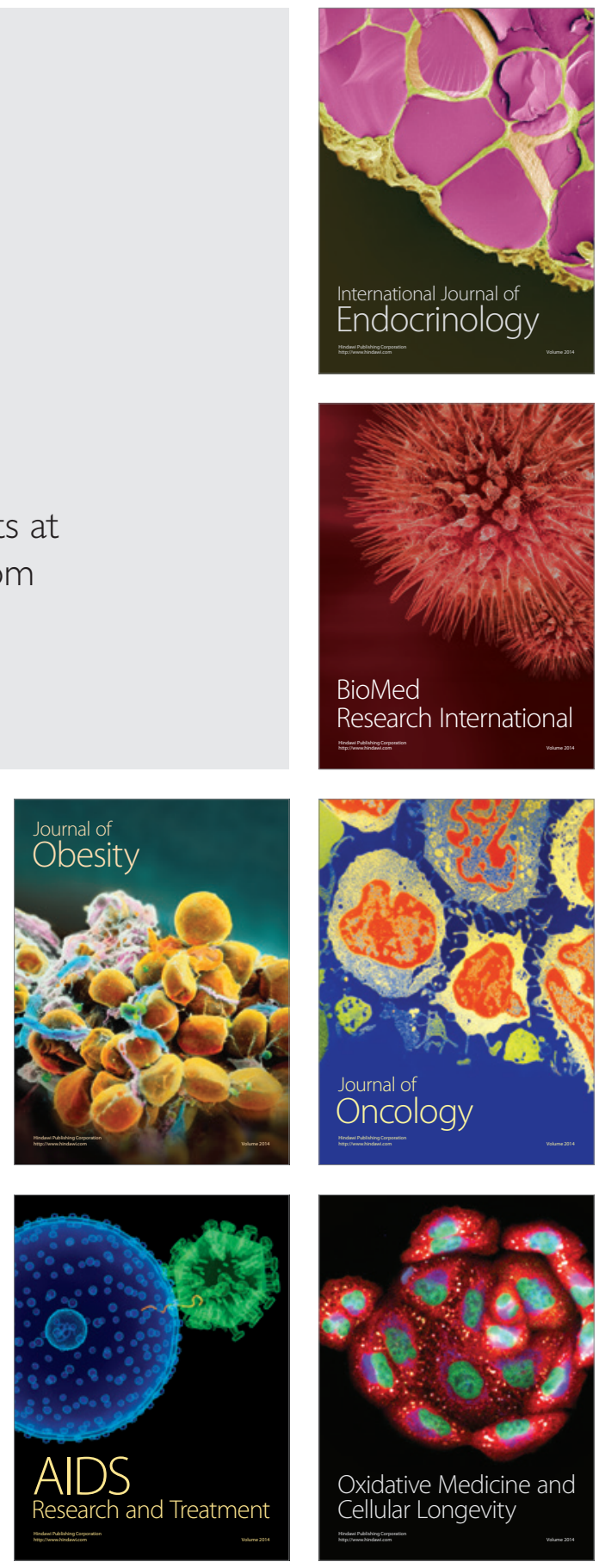